\title{
Advances in the Treatment of Cholinergic Anti-Inflammatory Pathways in Gastrointestinal Diseases by Electrical Stimulation of Vagus Nerve
}

\author{
Wei Leia, ${ }^{a}$ Zhijun Duan ${ }^{a, b}$ \\ aSecond Department of Gastroenterology, The First Affiliated Hospital of Dalian Medical University, Dalian, China; \\ ${ }^{b}$ Laboratory of Integrated Chinese and Western Medicine, The First Affiliated Hospital of Dalian Medical University, \\ Dalian, China
}

\section{Keywords}

Cholinergic anti-inflammatory pathway · Vagus nerve stimulation - a7 nicotinic acetylcholine receptor .

Gastrointestinal tract

\begin{abstract}
Background: The cholinergic anti-inflammatory pathway (CAIP) has been proposed as a key mechanism by which the brain, through the vagus nerve (VN), modulates the immune system in the body. Recent studies of VN stimulation (VNS) in vivo systems have shown that it plays an anti-inflammatory role through CAIP. Inflammatory diseases in the gastrointestinal tract are frequent and difficult to treat. Summary: The mechanism of the anti-inflammatory effect of VNS through CAIP is not fully known. The current review covers anatomy, molecular mechanisms, and the application in gastrointestinal diseases of the vagal CAIP. Key Messages: CAIP bridges immune and nervous systems and plays pleiotropic roles in modulating inflammation in animal models by targeting different immune, proinflammatory, epithelial and endothelial cells, and signaling pathways. Numerous animal studies have shown beneficial effects of stimulation of this pathway in models of inflammatory diseases, either through (electrical) stimulation of the VN or pharmacological approaches. In this review, we focus on the anti-inflammatory
\end{abstract}

benefits of VNS as a means of providing new insights into treating inflammation-related gastrointestinal diseases, as exemplified by those described herein. @ 2019 S. Karger AG, Basel

\section{Introduction}

Gastrointestinal diseases are common and many are associated with inflammation and some mainly dependent on drug treatment. In recent years, nondrug therapy has been highly praised for its fewer side effects. Vagus nerve (VN) stimulation (VNS) is one of the focuses, but the mechanism is not very clear. Cholinergic anti-inflammatory pathway (CAIP) has a restrictive effect on inflammation of the central nervous system (CNS). CNS pathways respond to injury or inflammation signals and reflexively inhibit inflammation cascades, which may occur in local and/or systemic inflammation $[1,2]$. In the inflammatory response, the peripheral and CNSs perceive inflammatory mediators and modulate downwardly through the typical efferent arm reflex. CAIP signals originate in the brainstem nucleus of the $\mathrm{VN}$ and pass through the VN to form synapses in the abdominal cavity and other peripheral ganglia [3]. According to the anatomical basis of the effect of the CAIP on the effector organs, the $\alpha 7$ karger@karger.com

www.karger.com/dig

(c) 2019 S. Karger AG, Basel

Karger"
Zhijun Duan

Second Department of Gastroenterology

The First Affiliated Hospital of Dalian Medical University

222 Zhongshan Road, Xigang, Dalian, Liaoning 0086-116011 (China)

E-Mail cathydoctor@sina.com 
nicotinic acetylcholine receptor ( $\alpha 7 \mathrm{nAChR})$ on the surface of macrophages in the vagal-innervated organs, such as liver, lung, and intestine, is activated by acetylcholine (ACh) released from the cholinergic nerve endings. The a7nAChR on macrophages, monocytes, and mast cells has been shown to mediate cholinergic anti-inflammatory output $[4,5]$. Some researchers have found that VNS achieves the anti-inflammatory effect by inhibiting macrophage production of proinflammatory cytokines with an $\alpha 7 \mathrm{nAChR}$-dependent manner [6]. The present paper will review the recent progress of CAIP in the treatment of gastrointestinal diseases by VNS.

As a treatment, VNS has attracted extensive attention, and its application in gastrointestinal diseases is gradually expanding. The VNS includes electrical stimulation (EVNS), chemical stimulation, acupuncture points, and so on. In the treatment of VNS, the anti-inflammatory effect is the most important. The anti-inflammatory pathway of $\mathrm{VN}$ not only takes a role in brain-gut-liver axis but also mediates the regulation of neuroimmunity. The further study of this neuroregulatory mechanism will provide more perspectives for the treatment of gastrointestinal diseases and lay a foundation for the finding of new therapeutic targets for intestinal immune diseases.

\section{Cholinergic Anti-Inflammatory Pathway}

\section{Anatomy}

As a bidirectional connection between the brain and the immune system, $\mathrm{VN}$ can reduce the inflammatory deterioration process outside the CNS [7]. Cytokines and/ or endotoxins can be detected in circulation when systemic inflammation occurs. Animal researches have clearly shown that the brain can perceive the presence of cytokines and/or endotoxins in the area postrema, the organum vasculum of the lamina terminalis, and the subfornical organ [8]. Under the condition of local peripheral inflammation, circumventricular organs cannot be activated because of the lack or too low level of cytokines. In this environment, the brain senses inflammation by introducing cytokine (IL-1) receptors on vagal afferents and glomus cells adjacent to the VN. In this "inflammatory reflex" mechanism, the increased release of local inflammatory cytokines activates vagal nerve fibers, which transmit signals from the vagal nerve fibers to the nucleus tractus solitarius (NTS) of the medulla oblongata. And NTS then projected to the efferent neurons of the dorsal motor nucleus of the $\mathrm{VN}$, further to the innate ganglion of the viscera [9].

EVNS Activates CAIP Associated with Gastrointestinal Disease
The VN covers almost the entire gastrointestinal tract. The nerve fibers of the nucleus ambiguous of the brainstem and the dorsal motor nucleus of the VN innervate the upper and middle digestive tracts, respectively, while the sacral nerve tract innervates the distal colon, rectum, and anal canal [10-14]. The efferent fibers from the dorsal vagal nucleus connect with the splenic nerve in the celiac mesenteric ganglion and transmit anti-inflammatory signals to the spleen. The splenic nerve endings release norepinephrine activates the adrenergic receptors of specific T lymphocytes (ChAT $+\mathrm{T}$ cells), which express choline acetyltransferase and synthesize ACh. The norepinephrine-activated CHAT + T cells enter splenic macrophages and release ACh. ACh released by ChAT $+\mathrm{T}$ cells activates $\alpha 7 \mathrm{nAChR}$ in macrophages, resulting in downregulation of proinflammatory cytokines production and release, while the level of anti-inflammatory cytokines remains unchanged [15]. In other words, instead of reaching the lamina propria directly, the vagus efferent fibers synapses act on intestinal neurons innervating the lamina propria, and then release $\mathrm{ACh}$ on the nicotinic or muscarinic receptors.

\section{$\alpha 7$ Nicotinic ACh Receptor}

Nicotine receptors are considered to be ligand-gated ion channel complexes. It is currently believed that $\mathrm{ACh}$ plays an anti-inflammatory role by binding to nicotine receptors to a large extent [16]. The muscarinic receptors and nicotine receptors both play an important role in lowering tumor necrosis factor (TNF) levels in endotoxemia, but the anti-inflammatory effect of $\mathrm{VN}$ on peripheral immune cells is unrelated to muscarinic receptors [17]. Therefore, the anti-inflammatory effects mediated by nicotinic receptors are mainly discussed.

The $\alpha 7 \mathrm{nAChR}$ is a homologous oligomer expressed extensively in the CNS and in addition to their ionic activity; these membrane proteins regulate several downstream signal cascades [18]. It transmits action electric potentials between cholinergic synapses by binding to the neurotransmitter ACh. $\alpha 7 \mathrm{nAChR}$ is essential for the cholinergic anti-inflammatory response and is expressed on the surface of many inflammatory cells such as macrophages, dendritic cells, B and T lymphocytes, polymorphonuclear neutrophils, endothelial cells, microglia, and mast cells [19-21].

The $\alpha 7 \mathrm{nAChR}$-mediated CAIP is associated with many diseases. Recently, Kong et al. [22] explored the protective effect of $\alpha 7 \mathrm{nAChR}$ on lipopolysaccharide (LPS)-induced cardiomyopathy by CAIP which reverses the pathological process of cardiomyopathy and reduces

Digestion 2021;102:128-138 
cardiomyocyte apoptosis. Yamada and Ichinose [23] found that $a 7 \mathrm{nAChR}$ also regulates lung inflammation and its activation inhibits cytokine synthesis in different immune cells, including respiratory cells. In addition, Hayashi et al. $[24,25]$ also found that $\alpha 7 n A C h R$ not only plays a role in lung inflammation but also may downregulate the innate immune system, regulate the defensive function of cancer cells, and affect the growth and secretion function of cancer cells. Ibrahim et al. [26] found that galantamine ( $\alpha 7 \mathrm{nAChR}$ agonist) can improve the survival rate of mice with acute kidney injury and improve the histopathological conditions. After $a 7 \mathrm{nAChR}$ is inhibited, this protective effect disappears, suggesting that this receptor also plays a significant role in the protection of renal inflammation. Moreover, $\alpha 7 \mathrm{nAChR}$ has anti-inflammatory effects in neurological diseases, such as ischemic encephalopathy and arthritis [27].

Among the gastrointestinal diseases, some scholars have demonstrated that macrophages are located near the nerve endings of gastric circular muscle and ileal plexus by double-labeled immunohistochemistry using vesicular ACh transporter-positive vagal efferent fibers and macrophages [28]. For example, Mihara et al. [29] found that the activation of $\alpha 7 \mathrm{nAChR}$ expressed in intestinal mesothelial cells reduced the expression of inflammatory factor IL-1 $\beta$. In the experimental colitis model, Salaga et al. [30] and Tasaka et al. [31] both indicated that $\alpha 7 \mathrm{nAChR}$ $\mathrm{R}$ could be activated to change the number or activation of intestinal immune cells to alleviate colitis. In terms of the improvement of postoperative intestinal obstruction (POI), Hong et al. [32] showed that the activation of a7nAChR on macrophages could inhibit intestinal wall inflammation and the development of POI.

\section{$\alpha 7 n A C h R$ Intracellular Signaling Mechanism}

After macrophage $\alpha 7 \mathrm{nAChR}$ activation, a series of intracellular signal changes may occur, leading to a decrease of inflammatory cytokines such as high-mobility group protein 1 (HMGB1) and TNF- $\alpha$. The current research focuses on 3 possible signal mechanisms (Fig. 1): First, by regulating the transcription factor $\mathrm{B}(\mathrm{NF}-\kappa \mathrm{B}), \alpha 7 \mathrm{nAChR}$ activating the expression of proinflammatory cytokines participates in the regulation of inflammatory processes. Second, activate the JAK2/STAT3 signaling cascade to regulate inflammatory responses. Finally, $\mathrm{Ca}^{2+}$-dependent mechanism activates classic PKC leading to increased production of reactive oxygen species and the activation of PI3K/Akt/Nrf-2 pathway, which induces the expression of heme oxygenase (HO-1) in macrophages to regulate the inflammatory response.

\section{NF- $\kappa B$ Pathways}

Other results showed that the activation of $\alpha 7 \mathrm{nAChR}$ in macrophages inhibited inflammation by inhibiting the nuclear translocation of NF- $\kappa B$ [22]. The regulation of $\mathrm{NF}-\kappa \mathrm{B}$ transcription factor on the inflammatory process is involved by activating the expression of proinflammatory cytokines [33]. Normally, NF- $\kappa$ B is inactivated in the cytoplasm and does not need to be stimulated, thus responding quickly to proinflammatory stimuli. NF- $\kappa B$ subunits P50 and p65 are inactivated by binding to I-kappa B [34]. When ligands such as proinflammatory cytokines and LPSs activate toll-like receptors 4, I-kappa B is phosphorylated by I-kappa $\kappa$ and degraded by the signal. With the phosphorylation of I-kappa B, P50 and P65 subunits of NF- $\kappa \mathrm{B}$ can be freely transported to the nucleus and activate the transcription of proinflammatory cytokines, thereby enlarging the body's inflammatory response to proinflammatory stimuli $[35,36]$. Therefore, inflammation can be regulated by regulating nuclear translocation of NF- $\kappa B$. In fact, the CAIP induced by activation of $\alpha 7 \mathrm{nAChR}$ prevents the nuclear translocation of NF- $\kappa B$ in macrophages, thus inhibiting the secretion of HMGB1, an important proinflammatory cytokine, which is a late inflammatory mediator in sepsis [37]. Besides, nicotine, as an agonist of $\alpha 7 \mathrm{nAChR}$, decreased serum HMGB1 level and improved the survival rate in sepsis experimental model. Nicotine also reduced TNF- $\alpha-$ induced nuclear translocation of macrophage NF- $\kappa \mathrm{B}$, increased the cytoplasmic levels of I-kappa a and $\varepsilon$, and the nicotine stimulation blocks cell activation and leukocyte recruitment during inflammation [38]. However, the exact mechanism of blocking nuclear translocation of $\mathrm{NF}-\kappa \mathrm{B}$ by activating $\alpha 7 \mathrm{nAChR}$ in macrophages remains unclear. Nevertheless, studies have shown that nicotine can induce IKK to inhibit the phosphorylation of I-kappa $\mathrm{B}$ and the transcriptional activity of NF- $\kappa \mathrm{B}$ [32]. Nicotine treatment has been also shown to increase STAT3 phosphorylation and reduce LPS-induced p65 translocation $[39,40]$.

\section{JAK2/STAT3}

Activating $a 7 \mathrm{nAChR}$ in macrophages can regulate inflammation by activating the JAK2/STAT3 signaling cascade. Anti-inflammatory cytokine IL-10 can activate the JAK2-STAT3 pathway [41]. The researchers found that nicotine had anti-inflammatory effects on peritoneal macrophages mediated by a $\mathrm{nAChR}$ and JAK2/STAT3 activation in vitro and in vivo. To clarify the role of the JAK2/STAT3 pathway in CAIP, 2 alternative models are proposed. 


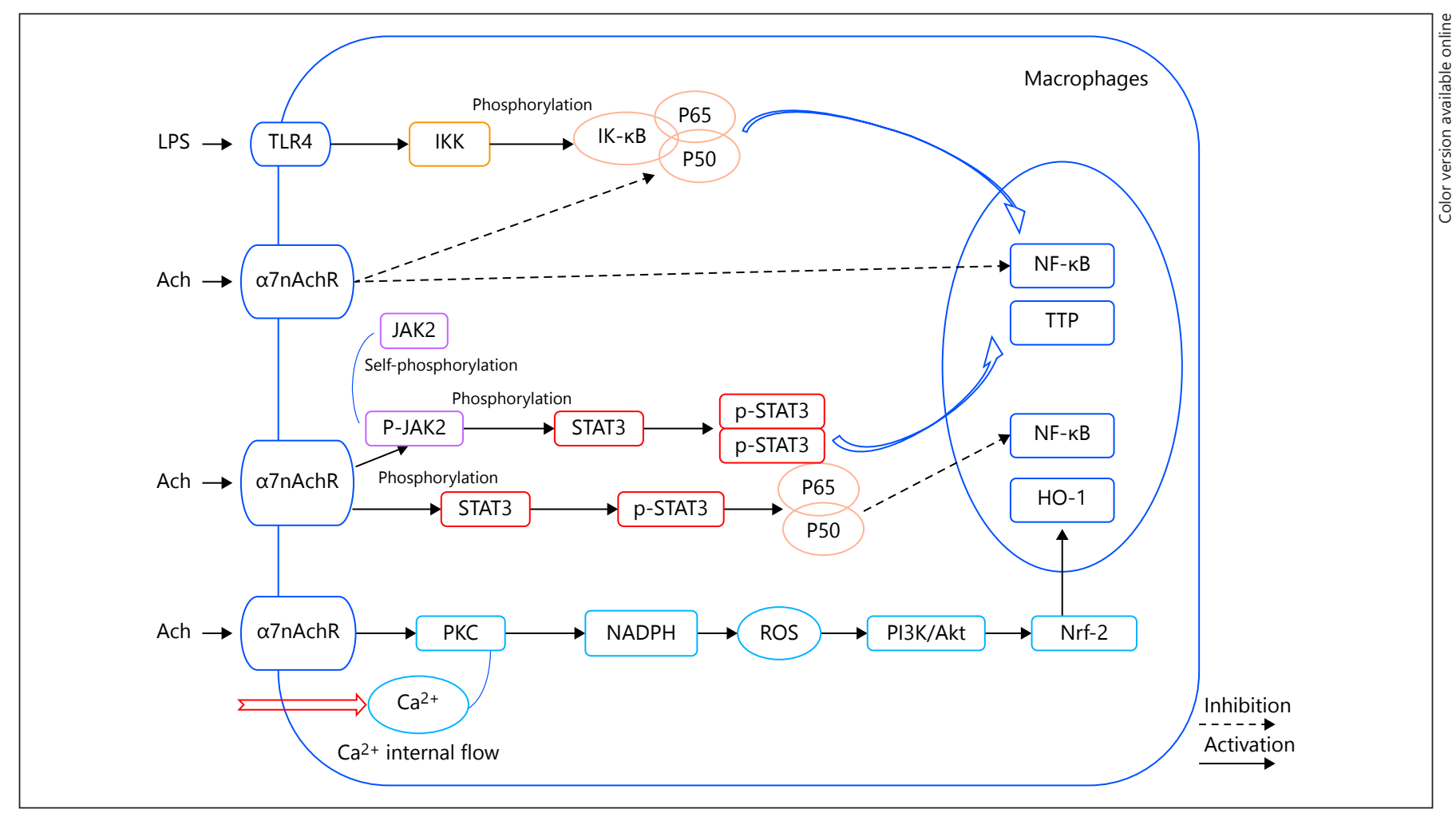

Fig. 1. The cholinergic anti-inflammatory reflex. (1) When ligands such as proinflammatory cytokines and lipopolysaccharides activate TLR4, IK-kappa B is phosphorylated by IKK and degraded by the signal. With the phosphorylation of I-kappa B, P50, and P65 subunits of NF- $\mathrm{KB}$ can be freely transported to the nucleus and activate the transcription of pro-inflammatory cytokines. The CAIP triggered by the activation of $\alpha 7 \mathrm{nAChR}$ prevented the nuclear translocation of NF- $\kappa B$ in macrophages and inhibited its transcriptional activity. (2) It has been reported that the activation of $\alpha 7 \mathrm{nAChR}$ can directly inhibit the transcriptional activity of NF- $\kappa B$. (3) JAK2 is autophosphorylated, then JAK2 aggregates and p-STAT3, and finally p-STAT3 forms dimer and transfers to the nucleus, p-STAT3 dimers have been found to block the production and release of proinflammatory cytokines, and another study

The first model suggests that the combination of cholinergic agonists with $\alpha 7 \mathrm{nAChR}$ leads to the recruitment of JAK2 to $\alpha 7 \mathrm{nAChR}$. JAK2 is autophosphorylated, and then JAK2 aggregates and phosphorylates STAT3 (p-STAT3), and finally p-STAT3 forms dimer and transfers to the nucleus [42-44]. Although STAT3 acts as a negative regulator of proinflammatory response, the mechanism of p-STAT3 dimer inhibiting the production and release of proinflammatory cytokines is not fully understood. A recent study has revealed that the mechanism of nicotine-activating STAT3 may be related to anti-inflammatory effects. In experiments using U937 cellmonocyte lines, nicotine-activated STAT3 has been showed that TTP is induced by activated STAT3, which may have anti-inflammatory effects. However, STAT3 may also interfere with LPS-induced proinflammatory response by binding to NF- $\kappa \mathrm{B}$ and form u-STAT3-NF- $\mathrm{uB}$ complex by replacing I-kappa $\mathrm{B}$. The u-STAT3-NF-kappa B complex can inhibit the activation of NF-kappa B and produce anti-inflammatory effect. (4) Another the possible mechanism regulating cholinergic anti-inflammation is the activation of PI3K/Akt/Nrf-2 pathway, which leads to the upregulation of HO-1. LPS, lipopolysaccharide; TLR4, toll-like receptors 4; Ach, acetylcholine; $\alpha 7 \mathrm{nAChR}, \alpha 7$ nicotinic acetylcholine receptor; NF- $\kappa \mathrm{B}$, transcription factor kappa B; TTP, tristetraprolin; p-STAT3, phosphorylates STAT3; HO-1, heme oxygenase; $\mathrm{NADPH}$, nicotinamide adenine dinucleotide phosphate; ROS, reactive oxygen species.

shown to induce tristetraprolin, an Au-rich-binding protein. The tristetraprolin induces cholinergic anti-inflammatory effects by destroying inflammatory transcripts containing Au-richs that are present in the $3^{\prime}$-untranslated region [45].

Peña et al. [46] proposed a second model for cholinergic regulation of inflammation associated with the JAK2/ STAT3 signaling cascade. They believe that unphosphorylated STAT3 (u-STAT3) has anti-inflammatory effects rather than $\mathrm{p}$-STAT3. The results suggested that the antiinflammatory response of cholinergic is due to the inhibition of STAT3 tyrosine phosphorylation. The results showed that the inhibition of STAT3 tyrosine phosphor- 
ylation was the main mechanism of the cholinergic antiinflammatory reaction. It is worth noting that nicotine was used as an agonist in previous articles that demonstrated the role of p-STAT3 in CAIP, which is different from Pena's experiment in which choline was used as an agonist. They believed that u-STAT3 replaced I-kappa B by combining with NF- $\kappa B$ to form a u-STAT3-NF- $\kappa B$ complex to alleviate LPS-induced inflammatory response. They hypothesize that the anti-inflammatory effect of cholinergic agonists produced by uSTAT3-NF- $\mathrm{BB}$ complex may be created through inhibiting the activation of NF- $\kappa$ B.

In addition to the above 2 models, another study showed that nonphosphorylated STAT3 might compete with NF- $\kappa$ B. The inhibition of STAT3 protein expression could enhance cytokine production and eliminate a7nAChR signaling [40]. Furthermore, other studies have found that nicotine increases the level of IL-1 receptor-related kinase $\mathrm{M}$ in macrophages, which depends on JAK2, STAT3, and PI3K [47].

\section{PI3K/Akt/Nrf-2}

Another mechanism of cholinergic regulation of proinflammatory response is mediated by the upregulation of HO-1. In neurosurgery and LPS administration models, the activation and oxidative stress of reduced nicotinamide adenine dinucleotide phosphate on monocytes are associated with neuroinflammation and memory dysfunction. The activation of NF-kappa B can reduce neuroinflammation and improve cognitive deficits, which activates the PI3K/Akt/Nrf-2 pathway [48, 49]. Oxidative stress and neuroinflammation are currently considered to be 2 of the most important pathological mechanisms in neurodegenerative diseases such as Alzheimer's disease, Parkinson's disease, and Huntington's chorea. It is also found that HO-1 expression is not related to the density of $\alpha 7 \mathrm{nAChR}$ in Huntington's chorea. Increased expression of HO-1 may induce neuroprotective effects related to the activation of $\alpha 7 \mathrm{nAChR}$ in early-stage Huntington's chorea by CAIP [50].

In experiments performed in RAW264.7 cells - a mouse mononuclear macrophage cell line - nicotine dose-dependent increased the expression of Ho- 1 through $\alpha 7 \mathrm{nAChR}$, and when $\alpha 7 \mathrm{nAChR}$ was antagonized by megaramine or silenced by RNA interference, the nicotineinduced Ho-1 upregulation was eliminated [51]. This study [51] confirms the necessity of upregulation of HO-1 induced by $\mathrm{Ca}^{2+}$ influx activation of $\alpha 7 \mathrm{nAChR}$ because the presence of $\mathrm{Ca}^{2+}$ chelators decreases the upregulation of HO-1 induced by nicotine, and the effect is dose-de- pendent. The activation of $\alpha 7 \mathrm{nAChR}$ is accompanied by $\mathrm{Ca}^{2+}$ influx and the termination of the anti-inflammatory cascade. Then the influx of $\mathrm{Ca}^{2+}$ activates the classical PKC pathway through $\mathrm{CA}^{2+}$-dependent mechanism. The oxidation of nicotinamide adenine dinucleotide phosphate oxidase is accompanied by the production of a large number of reactive oxygen species, and PI3K/Akt/Nrf-2 pathway of phosphoinositol-3 kinase was activated [52], which HO-1 expression in macrophages was further induced [53]. The nicotine-induced upregulation of HO-1 may play a necessary role in the anti-inflammatory effect of nicotine-induced macrophages.

\section{Vagus Nerve Electrical Stimulation}

In 1990, EVNS was first used in humans to treat drugresistant epilepsy [54]. Several years later, the US Food and Drug Administration approved EVNS for this indication and in 2005 for drug-resistant depression $[55,56]$. EVNS is a treatment that stimulates the VN with an implantable device, that is, spiral electrodes were wound on the $\mathrm{VN}$ in the neck through surgical operation, the stimulator is buried in the chest, the parameters and modes of the device are adjusted, so that the stimulator can automatically stimulate the $\mathrm{VN}$ for therapeutic purposes [57].

With the further study of VN, the application of EVNS involves more subjects, for example, EVNS can reduce the area of cerebral infarction and improve the neurological function score after cerebral ischemia $[58,59]$ and can also be used to treat heart failure, thereby increasing left ventricular ejection fraction and NYHA score [60].

Electroacupuncture (EA) is an improvement on the traditional EVNS, which improves the safety and tolerance of EVNS because it does not require surgical implantation. The traditional EVNS needs to implant a small electrode piece into the body, while EA only needs to needle specific parts or stick the electrode piece on specific parts. The traditional Chinese medicine acupuncture has a history of $>2000$ years and is becoming an epidemic therapy for various diseases worldwide [61, 62]. Acupuncture is a medical intervention in the process of which slender needles are penetrated into muscles or other subcutaneous tissues are acted on acupoints, specific parts of the body. The traditional medical theory holds that the stimulation of acupuncture promotes the flow of "Qi" in the body, thus promoting the circulation of meridians and collaterals and finally promoting blood circulation [5758]. Acupoints are considered to be pathophysiologically related to visceral and systemic conditions and may reflect 
the state of visceral organs and the state of the whole body. Therefore, the stimulation of specific acupoints can cause reactivity, thereby controlling the imbalance of the internal environment and improving physical symptoms [63, 64]. Acupuncture stimulation is the direct treatment of local symptoms at or near acupoints, such as knee pain or muscle stiffness, at acupoints or adjacent affected areas, whereas acupuncture stimulation at distant affected areas is used to treat visceral diseases and systemic abnormalities. In EA treatment, a pair of needles was applied with a small current, and studies have shown that the therapeutic effect of EA can be regulated by changing the frequency, intensity, and duration of electrical stimulation $[65,66]$. For example, EA can activate different opioid receptors at low and high frequencies to achieve different analgesic effects $[67,68]$. Besides, it has the function of bidirectionally regulating the neuroendocrine-immune system, which can antagonize systemic inflammation without side effects.

However, the mechanism of EA treatment on gastrointestinal diseases is not completely clear and needs further study. EA has been reported to induce the $\mathrm{VN}$ to activate the aromatic amino acid decarboxylase, which leads to the production of dopamine in the adrenal medulla, thus controlling systemic inflammatory response $[69,70]$. At present, in gastrointestinal diseases, the most relevant point is Zusanli (ST36), Zusanli point belongs to the stomach meridian of Zuyangming. It is also the combination point of the stomach meridian, one of the 9 points of the lower stomach and Huiyang, the key point of strength. Zusanli is named for its ability to treat upper, middle, and lower abdominal diseases. It is found that acupuncture at ST36 is related to the parasympathetic nervous system, which can alleviate inflammation and promote gastrointestinal function $[71,72]$. Since the effect of acupuncture on ST36 is similar to that of activating the $\mathrm{VN}$, it is possible to have a positive effect on gastrointestinal diseases by stimulating the anti-inflammatory pathway of the VN.

\section{EVNS and Gastrointestinal Diseases}

\section{Postoperative Intestinal Obstruction}

Every patient undergoing abdominal surgery suffers from temporary impaired gastrointestinal motility or POI. TNF- $\alpha$ released from inflammatory muscular lamina propria and activated permanent macrophages following abdominal surgery [73]. Exhaustion and inactivation of macrophages in the muscular layer can prevent POI.
During operation, EVNS can reduce the inflammatory response to intestinal operation [74]. This anti-inflammatory effect mediated by macrophage activation and cytokine production reduction is driven by CAIP [75]. EVNS may be a potential therapy for POI prevention because CAIP as a therapeutic target can improve POI through its anti-inflammatory effect.

Stakenborg et al. [76] showed in preclinical models that preoperative VNS could reduce inflammation caused by surgery and prevent POI. It was also confirmed that this anti-inflammatory effect was caused by ACh acting on $a 7 \mathrm{nAChR}$ located in macrophages. Stakenborg et al. [77] stimulated abdominal VN in POI mice and found that LPS-induced TNF- $\alpha$ levels decreased, while significantly improved intestinal transport. They also conducted clinical trials in humans and found that abdominal VNS significantly reduced the production of whole blood IL-8 and IL- 6 induced by LPS.

EA has also been shown to improve symptoms of POI. Hong et al. [78] tested whether noninvasive auricular electrical percutaneous vagus stimulation affects inflammation in POI models. The results showed that EA activated NTS and DMV, decreased the expression of intestinal cytokines, and reduced the recruitment of white blood cells to the intestinal segment of the operation, which improved the gastrointestinal transport after an operation. Clinical prospective studies were also conducted, to provide evidence for EA activation of the efferent visceral VN fibers. It is proved that EA is a low risk, simple operation and can effectively prevent POI after an operation. Zhang et al. [79] also recruited 42 patients who were about to undergo abdominal surgery in clinical trials and conducted ST36 electrical stimulation, which further showed that the main symptoms after surgery could be improved by enhancing vagal activity and inhibiting sympathetic activity.

\section{Inflammatory Bowel Disease}

Inflammatory bowel disease (IBD) is a chronic inflammatory disease with an increasing incidence worldwide. IBD is generally divided into 2 types, namely, Crohn's disease and ulcerative colitis. Low vagal activity has been observed in IBD disease, and thus the relationship between IBD and VN has also been gradually recognized. Experimental evidence in mouse models suggests that the reflex control of intestinal inflammation is mediated by efferent vagal nerve signals. This signal is transmitted to macrophages located in the intestinal tract and mediated by an inflammatory reflex, through a nerve-immune interaction in the spleen $[80,81]$. 
Animal experiments have shown that the use of oxazolidone in the colon can seriously damage the colon mucosa, resulting in a mortality rate of $65 \%$ on the fifth day [82]. Compared with sham-treated mice, EVNS significantly increased the survival rate of model mice, which was related to the decrease of HMGB1 and the decrease of cytokine levels in colon and serum. After EVNS treatment in the TNBS-induced colitis model, colitis was alleviated compared with the control group by evaluating physiological (such as weight, temperature, exercise activity), macroscopic (lesion area), histological, and biological characteristics (such as cytokine and cytokine-related mRNAs) [83]. In the experiment with EVNS applied by Meregnani et al. [84] in the rat model, the course of colitis induced by TNBS was relieved, which was related to the inhibition of nuclear translocation of NF-kappa B and the upregulation of mitogen-activated protein kinase, both of which were important factors of inflammation. Sun et al. [85]. also used clinical, histological, and biochemical parameters to assess the chronic EVNS effect and recorded the heart rate variability in rats with colitis using EVNS. They also observed a significant decrease in colon inflammation and IL- 6 and TNF- $\alpha$ cytokines stimulated by the VN. Subsequently, Jin et al. [66] used a similar method and the same model of TNBS colitis, suggesting that chronic VNS can improve colonic inflammation by inhibiting proinflammatory cytokines through an autonomous mechanism.

In IBD, clinical studies using EVNS are limited. Two clinical studies have shown preliminary clinical outcomes of EVNS in patients with active CD. Bonaz et al. [86] reported a 6-month follow-up study of $7 \mathrm{CD}$ patients, 2 of whom were receiving azathioprine treatment, and the remaining 5 of whom were not receiving treatment at the same time. Before treatment, the endoscopic inflammation score (CDEIS) of Crohn's disease was $>7$ in all patients. The Crohn's disease activity index and CDEIS were evaluated after 6 months of continuous treatment. Two patients withdrew from the study in advance because of the deterioration of their condition. The activity index of Crohn's disease was improved in the remaining 5 patients, and clinical remission was achieved in 4 of them at the end of the study (Crohn's disease activity index $<150$ ). Within 6 months, all patients achieved endoscopic remission (CDEIS $<6$ ). All 5 patients reported reduced pain after 6 months. EVNS can also be used to maintain drug-induced remission. As a slow-acting treatment, EVNS is an effective means to prevent recurrence after CD surgery.

\section{EVNS and Intestinal Barrier}

The intestinal barrier consists of the mechanical barrier, chemical barrier, microbial barrier, and immune barrier. Intestinal mucosal barrier injury involves many fields such as microecology, immunity, and molecular biology. Intestinal epithelial dysfunction is associated with the development of various gastrointestinal diseases, such as Bauer's syndrome, IBD, abdominal diseases, and mucosal diseases [87-89].

Studies have shown that cutting the abdominal VN at the gastroesophageal junction before EVNS prevents the protective effect of EVNS, thus confirming the efferent $\mathrm{VN}$ signal could regulate the integrity of intestinal barrier after injury [90]. The intestinal permeability of animals with abdominal vagotomy before EVNS is comparable to that of animals with burns. Abdominal vagotomy eliminates the protective effect of EVNS. They also showed that the protective effect of EVNS on the intestinal tract was not due to the regulation of TNF- $\alpha$ production in splenic circulation. It is well known that intestinal glia can improve intestinal barrier function by increasing the expression of tight junction protein, thus improving the integrity of the intestinal barrier. The barrier induction of enteral glial activation can prevent or limit the disruption of the intestinal barrier, which may be a therapeutic target for diseases that cause intestinal inflammation. Costantini et al. [91] further demonstrated that $\alpha 7 n A C h R$ is a necessary receptor for the vagal nerve to regulate inflammation and mediate the protective effect of the intestinal barrier in the burn model.

The role of EA in intestinal barrier protection has also been discussed. Hu et al. [92] studied the protective effect and mechanism of vagal nerve stimulation on intestinal barrier dysfunction and distal organ injury after intestinal ischemia-reperfusion injury in rats utilizing integrated traditional Chinese and Western medicine. They compared the effects of EA at ST36 and non-acupoint points, as well as intraperitoneal injection of cholinergic agonists. The levels of cytokines in plasma, intestinal tissue, lung tissue, and liver tissue were determined. Intestinal barrier damage was detected by histology, intestinal injury score, the permeability of $4 \mathrm{kDa}$-fitc dextran, and changes of tight junction protein $\mathrm{ZO}-1$ detected by immunofluorescence and Western blot. The results showed that EA can significantly reduce the levels of TNF and IL-8 in plasma and organ tissues, decrease intestinal permeability of fitc-dextran, and prevent the expression and localization of ZO-1 protein. However, abdominal vagot- 
omy or intraperitoneal injection of $\alpha 7 \mathrm{nAChR}$ inhibitors reversed these effects.

All these results suggest that in the presence of intact $\mathrm{VN}$, EVNS can protect the integrity of the intestinal barrier and reduce the systemic inflammatory response and distant organ damage.

\section{Conclusion}

VN plays an anti-inflammatory role through the CAIP, targeting the $\mathrm{VN}$, which opens up a new therapeutic approach for gastrointestinal inflammatory diseases (such as IBD, POI, IBS) and other TNF- $\alpha$-mediated diseases (such as RA or psoriasis). Although the exact mechanism and neural circuits of intestinal CAIP have not been fully clarified, many experiments have shown that vagal nerve stimulation by electrical or chemical stimulation can alleviate inflammation. We gradually recognized how the $\mathrm{VN}$ is activated by certain inflammatory conditions and inhibits the production of proinflammatory cytokines through the activation of macrophage $a 7 \mathrm{nAChR}$. However, the existing research results still do not fully explain how the VN affects and controls inflammation in the gastrointestinal tract. From the perspective of anatomy, abdominal VN does not directly contact the intestinal im- mune cells, but the macrophages are located near the nerve endings of the gastric circular muscle and ileal plexus. The brain-gut axis from nerve to intestine needs further exploration and research.

Artificial modulation of peripheral nerve signals by VNS (neuromodulation) is an innovative therapy with the potential to develop alternative or complementary drug therapy. More clinical studies are needed to confirm its feasibility, which will lay the foundation for the longterm use of nondrug and noninvasive therapies to treat diseases and may become an important treatment for inflammation-related diseases shortly.

\section{Disclosure Statement}

The authors have no conflicts of interest to disclose.

\section{Funding Sources}

No funding was received for this study.

\section{Author Contributions}

W.L. wrote the paper. All authors read and gave approval of the final version of the article to be published.

\section{References}

1 Goverse G, Stakenborg M, Matteoli G. The intestinal cholinergic anti-inflammatory pathway. J Physiol. 2016 Oct;594(20):577180.

2 Carnevale D, Perrotta M, Pallante F, Fardella V, Iacobucci R, Fardella S, et al. A cholinergicsympathetic pathway primes immunity in hypertension and mediates brain-to-spleen communication. Nat Commun. 2016 Sep; 7(1):13035

3 Downs AM, Bond CE, Hoover DB. Localization of $\alpha 7$ nicotinic acetylcholine receptor mRNA and protein within the cholinergic anti-inflammatory pathway. Neuroscience. 2014 Apr;266:178-85.

4 Meroni E, Stakenborg N, Viola MF, Boeckxstaens GE. Intestinal macrophages and their interaction with the enteric nervous system in health and inflammatory bowel disease. Acta Physiol (Oxf). 2019 Mar;225(3):e13163.

5 Yamamoto T, Kodama T, Lee J, Utsunomiya N, Hayashi S, Sakamoto H, et al. Anti-allergic role of cholinergic neuronal pathway via $a 7$ nicotinic ACh receptors on mucosal mast cells in a murine food allergy model. PLoS One. 2014 Jan;9(1):e85888.
6 Hoover DB. Cholinergic modulation of the immune system presents new approaches for treating inflammation. Pharmacol Ther. 2017 Nov;179:1-16.

7 Borges GR, Morgan DA, Ketsawatsomkron P, Mickle AD, Thompson AP, Cassell MD, et al. Interference with peroxisome proliferatoractivated receptor- $\gamma$ in vascular smooth muscle causes baroreflex impairment and autonomic dysfunction. Hypertension. 2014 Sep; 64(3):590-6.

8 Matteoli G, Boeckxstaens GE. The vagal innervation of the gut and immune homeostasis. Gut. 2013 Aug;62(8):1214-22.

9 Trakhtenberg EF, Goldberg JL. Immunology. Neuroimmune communication. Science. 2011 Oct;334(6052):47-8.

10 Neuhuber WL, Kressel M, Stark A, Berthoud HR. Vagal efferent and afferent innervation of the rat esophagus as demonstrated by anterograde DiI and DiA tracing: focus on myenteric ganglia. J Auton Nerv Syst. 1998 May; 70(1-2):92-102.

11 Bautista TG, Sun QJ, Zhao WJ, Pilowsky PM. Cholinergic inputs to laryngeal motoneurons functionallyidentified in vivo in rat:a combined electrophysiological and microscopic study. J Comp Neurol. 2010 Dec;518(24):4903-16.

12 Berthoud HR, Carlson NR, Powley TL. Topography of efferent vagal innervation of the rat gastrointestinal tract. Am J Physiol. 1991 Jan;260(1 Pt 2):R200-7.

13 Schemann M, Grundy D. Electrophysiological identification of vagally innervated enteric neurons in guinea pig stomach. Am J Physiol. 1992 Nov;263(5 Pt 1):G709-18.

14 Berthoud HR, Jedrzejewska A, Powley TL. Simultaneous labeling of vagal innervation of the gut and afferent projections from the visceral forebrain with dil injected into the dorsal vagal complex in the rat. J Comp Neurol. 1990 Nov;301(1):65-79.

15 Borovikova LV, Ivanova S, Zhang M, Yang $\mathrm{H}$, Botchkina GI, Watkins LR, et al. Vagus nerve stimulation attenuates the systemic inflammatory response to endotoxin. Nature. 2000 May;405(6785):458-62.

16 Gotti C, Clementi F, Fornari A, Gaimarri A, Guiducci S, Manfredi I, et al. Structural and functional diversity of native brain neuronal nicotinic receptors. Biochem Pharmacol. 2009 Oct;78(7):703-11. 
17 Pavlov VA, Ochani M, Gallowitsch-Puerta M, Ochani K, Huston JM, Czura CJ, et al. Central muscarinic cholinergic regulation of the systemic inflammatory response during endotoxemia. Proc Natl Acad Sci USA. 2006 Mar; 103(13):5219-23.

18 Stokes C, Treinin M, Papke RL. Looking below the surface of nicotinic acetylcholine receptors. Trends Pharmacol Sci. 2015 Aug; 36(8):514-23.

19 Cormier A, Paas Y, Zini R, Tillement JP, Lagrue G, Changeux JP, et al. Long-term exposure to nicotine modulates the level and activity of acetylcholine receptors in white blood cells of smokers and model mice. Mol Pharmacol. 2004 Dec;66(6):1712-8.

20 Sato KZ, Fujii T, Watanabe Y, Yamada S, Ando T, Kazuko F, et al. Diversity of mRNA expression for muscarinic acetylcholine receptor subtypes and neuronal nicotinic acetylcholine receptor subunits in human mononuclear leukocytes and leukemic cell lines. Neurosci Lett. 1999 Apr;266(1):17-20.

21 Ransohoff RM, Cardona AE. The myeloid cells of the central nervous system parenchyma. Nature. 2010 Nov;468(7321):253-62.

22 Kong W, Kang K, Gao Y, Liu H, Meng X, Cao Y, et al. GTS-21 Protected Against LPS-Induced Sepsis Myocardial Injury in Mice Through $\alpha 7 \mathrm{nAChR}$. Inflammation. 2018 Jun; 41(3):1073-83.

23 Yamada M, Ichinose $M$. The cholinergic antiinflammatory pathway: an innovative treatment strategy for respiratory diseases and their comorbidities. Curr Opin Pharmacol. 2018 Jun;40:18-25.

24 Hayashi S, Hamada T, Zaidi SF, Oshiro M, Lee J, Yamamoto T, et al. Nicotine suppresses acute colitis and colonic tumorigenesis associated with chronic colitis in mice. Am J Physiol Gastrointest Liver Physiol. 2014 Nov; 307(10):G968-78.

25 Berne S, Čemažar M, Frangež R, Juntes $\mathrm{P}$, Kranjc S, Grandič M, et al. APS8 Delays Tumor Growth in Mice by Inducing Apoptosis of Lung Adenocarcinoma Cells Expressing High Number of a7 Nicotinic Receptors. Mar Drugs. 2018 Oct;16(10):E367.

26 Ibrahim SM, Al-Shorbagy MY, Abdallah DM, El-Abhar HS. Activation of a7 Nicotinic Acetylcholine Receptor Ameliorates ZymosanInduced Acute Kidney Injury in BALB/c Mice. Sci Rep. 2018 Nov;8(1):16814.

27 Li X, Huang Z, Liu S, Zeng X, Xie J, Liu C, et al. $3^{\prime}$-Daidzein sulfonate sodium provides neuroprotection by promoting the expression of the $\alpha 7$ nicotinic acetylcholine receptor and suppressing inflammatory responses in a rat model of focal cerebral ischemia. Am J Transl Res. 2018 Nov;10(11): 3455-64.

28 de Jonge WJ, van der Zanden EP, The FO, Bijlsma MF, van Westerloo DJ, Bennink RJ, et al. Stimulation of the vagus nerve attenuates macrophage activation by activating the Jak2STAT3 signaling pathway. Nat Immunol. 2005 Aug;6(8):844-51.
29 Mihara T, Otsubo W, Horiguchi K, Mikawa S, Kaji N, Iino S, et al. The anti-inflammatory pathway regulated via nicotinic acetylcholine receptors in rat intestinal mesothelial cells. J Vet Med Sci. 2017 Nov;79(11):1795802.

30 Salaga M, Blomster LV, Piechota-Polańczyk A, Zielińska M, Jacenik D, Cygankiewicz AI, et al. Encenicline, an $\alpha 7$ nicotinic acetylcholine receptor partial agonist, reduces immune cell infiltration in the colon and improves experimental colitis in mice. J Pharmacol Exp Ther. 2016 Jan;356(1):157-69.

31 Tasaka Y, Yasunaga D, Kiyoi T, Tanaka M, Tanaka A, Suemaru K, et al. Involvement of stimulation of $\alpha 7$ nicotinic acetylcholine receptors in the suppressive effect of tropisetron on dextran sulfate sodium-induced colitis in mice. J Pharmacol Sci. 2015 Mar;127(3):27583.

32 Hong GS, Zillekens A, Schneiker B, Pantelis $\mathrm{D}$, de Jonge WJ, Schaefer N, et al. Non-invasive transcutaneous auricular vagus nerve stimulation prevents postoperative ileus and endotoxemia in mice. Neurogastroenterol Motil. 2019 Mar;31(3):e13501.

33 Lu Z, Xie P, Zhang D, Sun P, Yang H, Ye $J$, et al. 3-Dehydroandrographolide protects against lipopolysaccharide-induced inflammation through the cholinergic anti-inflammatory pathway. Biochem Pharmacol. 2018 Dec;158:305-17.

34 Wang $\mathrm{H}$, Liao H, Ochani M, Justiniani M, Lin $\mathrm{X}$, Yang L, et al. Cholinergic agonists inhibit HMGB1 release and improve survival in experimental sepsis. Nat Med. 2004 Nov;10(11): 1216-21.

35 Yue Y, Liu R, Cheng W, Hu Y, Li J, Pan X, et al. GTS-21 attenuates lipopolysaccharide-induced inflammatory cytokine production in vitro by modulating the Akt and NF- $\mathrm{kB}$ signaling pathway through the $\alpha 7$ nicotinic acetylcholine receptor. Int Immunopharmacol. 2015 Dec;29(2):504-12.

36 Zhang M, Han X, Bao J, Yang J, Shi SQ, Garfield RE, et al. Choline Supplementation During Pregnancy Protects Against Gestational Lipopolysaccharide-Induced Inflammatory Responses. Reprod Sci. 2018 Jan;25(1):74-85.

37 Xu D, Lv Y, Wang J, Yang M, Kong L. Deciphering the mechanism of Huang-Lian-Jie$\mathrm{Du}$-Decoction on the treatment of sepsis by formula decomposition and metabolomics: enhancement of cholinergic pathways and inhibition of HMGB-1/TLR4/NF- $\kappa B$ signaling. Pharmacol Res. 2017 Jul;121:94-113.

38 Sánchez-Alemán E, Quintanar-Stephano A, Escobedo G, Campos-Esparza MR, CamposRodríguez R, Ventura-Juárez J. Vagotomy induces deregulation of the inflammatory response during the development of amoebic liver abscess in hamsters. Neuroimmunomodulation. 2015;22(3):166-80.

39 Kong W, Kang K, Gao Y, Liu H, Meng X, Yang S, et al. Dexmedetomidine alleviates LPS-induced septic cardiomyopathy via the cholinergic anti-inflammatory pathway in mice. Am J Transl Res. 2017 Nov;9(11):50407.

40 Sui HX, Ke SZ, Xu DD, Lu NN, Wang YN, Zhang YH, et al. Nicotine induces TIPE2 upregulation and Stat3 phosphorylation contributes to cholinergic anti-inflammatory effect. Int J Oncol. 2017 Sep;51(3):987-95.

41 Zhao M, He X, Bi XY, Yu XJ, Gil Wier W, Zang WJ. Vagal stimulation triggers peripheral vascular protection through the cholinergic anti-inflammatory pathway in a rat model of myocardial ischemia/reperfusion. Basic Res Cardiol. 2013 May;108(3):345.

42 Zhang XF, Xiang SY, Geng WY, Cong WJ, Lu J, Jiang CW, et al. Electro-acupuncture regulates the cholinergic anti-inflammatory pathway in a rat model of chronic obstructive pulmonary disease. J Integr Med. 2018 Nov; 16(6):418-26.

43 Park H, Park H, Mun D, Kim M, Pak HN, Lee $\mathrm{MH}$, et al. Sympathetic nerve blocks promote anti-inflammatory response by activating the JAK2-STAT3-mediated signaling cascade in rat myocarditis models: A novel mechanism with clinical implications. Heart Rhythm. 2018 May;15(5):770-9.

44 Li T, Wu S, Zhang H, Wang Y, Luo H, Zuo X, et al. Activation of Nicotinic Receptors Inhibits TNF- $\alpha$-Induced Production of Pro-inflammatory Mediators Through the JAK2/ STAT3 Signaling Pathway in Fibroblast-Like Synoviocytes. Inflammation. 2015 Aug;38(4): 1424-33.

45 Joe Y, Kim HJ, Kim S, Chung J, Ko MS, Lee $\mathrm{WH}$, et al. Tristetraprolin mediates anti-inflammatory effects of nicotine in lipopolysaccharide-stimulated macrophages. J Biol Chem. 2011 Jul;286(28):24735-42.

46 Peña G, Cai B, Liu J, van der Zanden EP, Deitch EA, de Jonge WJ, et al. Unphosphorylated STAT3 modulates alpha 7 nicotinic receptor signaling and cytokine production in sepsis. Eur J Immunol. 2010 Sep;40(9):2580-9.

47 Maldifassi MC, Atienza G, Arnalich F, LópezCollazo E, Cedillo JL, Martín-Sánchez C, et al. A new IRAK-M-mediated mechanism implicated in the anti-inflammatory effect of nicotine via $\alpha 7$ nicotinic receptors in human macrophages. PLoS One. 2014 Sep;9(9):e108397.

48 Terrando N, Yang T, Ryu JK, Newton PT, Monaco C, Feldmann M, et al. Stimulation of the $\alpha 7$ nicotinic acetylcholine receptor protects against neuroinflammation after tibia fracture and endotoxemia in mice. Mol Med. 2015 Mar;20(1):667-75.

49 Egea J, Buendia I, Parada E, Navarro E, León $\mathrm{R}$, Lopez MG. Anti-inflammatory role of microglial alpha7 nAChRs and its role in neuroprotection. Biochem Pharmacol. 2015 Oct; 97(4):463-72.

50 Foucault-Fruchard L, Tronel C, Bodard S, Gulhan Z, Busson J, Chalon S, et al. Alpha-7 nicotinic acetylcholine receptor agonist treatment in a rat model of Huntington's disease and involvement of heme oxygenase-1. Neural Regen Res. 2018 Apr;13(4): $737-41$. 
51 Tsoyi K, Jang HJ, Kim JW, Chang HK, Lee YS, Pae HO, et al. Stimulation of alpha7 nicotinic acetylcholine receptor by nicotine attenuates inflammatory response in macrophages and improves survival in experimental model of sepsis through heme oxygenase-1 induction. Antioxid Redox Signal. 2011 Jun;14(11): 2057-70.

52 Rehani K, Scott DA, Renaud D, Hamza H, Williams LR, Wang $\mathrm{H}$, et al. Cotinine-induced convergence of the cholinergic and PI3 kinase-dependent anti-inflammatory pathways in innate immune cells. Biochim Biophys Acta. 2008 Mar;1783(3):375-82.

53 Park J, Kang JW, Lee SM. Activation of the cholinergic anti-inflammatory pathway by nicotine attenuates hepatic ischemia/reperfusion injury via heme oxygenase-1 induction. Eur J Pharmacol. 2013 May;707(1-3):61-70.

54 Penry JK, Dean JC. Prevention of intractable partial seizures by intermittent vagal stimulation in humans: preliminary results. Epilepsia. 1990;31(suppl 2):S40-3.

55 Rush AJ, Marangell LB, Sackeim HA, George MS, Brannan SK, Davis SM, et al. Vagus nerve stimulation for treatment-resistant depression: a randomized, controlled acute phase trial. Biol Psychiatry. 2005 Sep;58(5):347-54.

56 Rush AJ, Sackeim HA, Marangell LB, George MS, Brannan SK, Davis SM, et al. Effects of 12 months of vagus nerve stimulation in treatment-resistant depression: a naturalistic study. Biol Psychiatry. 2005 Sep;58(5):35563.

57 Reid SA. Surgical technique for implantation of the neurocybernetic prosthesis. Epilepsia. 1990;31(Suppl 2):S38-9.

58 Guan YZ, Jin XD, Guan LX, Yan HC, Wang $\mathrm{P}$, Gong $\mathrm{Z}$, et al. Nicotine inhibits microglial proliferation and is neuroprotective in global ischemia rats. Mol Neurobiol. 2015;51(3): $1480-8$.

59 Ay I, Lu J, Ay H, Gregory Sorensen A. Vagus nerve stimulation reduces infarct size in rat focal cerebral ischemia. Neurosci Lett. 2009 Aug;459(3):147-51.

60 Zhang Y, Popovic ZB, Bibevski S, Fakhry I, Sica DA, Van Wagoner DR, et al. Chronic vagus nerve stimulation improves autonomic control and attenuates systemic inflammation and heart failure progression in a canine high-rate pacing model. Circ Heart Fail. 2009 Nov;2(6):692-9.

61 Shi P, Sun LL, Lee YS, Tu Y. Electroacupuncture regulates the stress-injury-repair chain of events after cerebral ischemia/reperfusion injury. Neural Regen Res. 2017 Jun;12(6):92530.

62 Park JY, Kim YK, Kim SY, Lee H, Choi CJ, Chae Y, et al. Acupuncture modulates brain neural activity in patients: a systematic review and meta-analysis. Orient Pharm Exp Med. 2017;17(2):111-26.

63 World Health Organization. WHO International Standard Terminologies on Traditional Medicine in the Western Pacific Region. Geneva: WHO; 2007.
64 Stux G, Pomeranz B. Acupuncture: Textbook and Atlas. Heidelberg: Springer; 2012.

65 Ma W, Li Z, Lu Z, Tan W, Zhang Z, Li Y, et al. Protective effects of acupuncture in cardiopulmonary bypass-induced lung injury in rats. Inflammation. 2017 Aug;40(4):1275-84.

66 Jin H, Guo J, Liu J, Lyu B, Foreman RD, Yin J, et al. Anti-inflammatory effects and mechanisms of vagal nerve stimulation combined with electroacupuncture in a rodent model of TNBS-induced colitis. Am J Physiol Gastrointest Liver Physiol. 2017 Sep;313(3):G192202.

67 Zhang R, Lao L, Ren K, Berman BM. Mechanisms of acupuncture-electroacupuncture on persistent pain. Anesthesiology. 2014 Feb; 120(2):482-503.

68 Lin JG, Chen WL. Acupuncture analgesia: a review of its mechanisms of actions. Am J Chin Med. 2008;36(4):635-45.

$69 \mathrm{Du}$ MH, Luo HM, Hu S, Lv Y, Lin ZL, Ma L. Electroacupuncture improves gut barrier dysfunction in prolonged hemorrhagic shock rats through vagus anti-inflammatory mechanism. World J Gastroenterol. 2013 Sep; 19(36):5988-99.

70 Torres-Rosas R, Yehia G, Peña G, Mishra P, del Rocio Thompson-Bonilla M, Moreno-Eutimio MA, et al. Dopamine mediates vagal modulation of the immune system by electroacupuncture. Nat Med. 2014 Mar;20(3): 291-5.

71 Iwa M, Matsushima M, Nakade Y, Pappas TN, Fujimiya M, Takahashi T. Electroacupuncture at ST-36 accelerates colonic motility and transit in freely moving conscious rats. Am J Physiol Gastrointest Liver Physiol. 2006 Feb;290(2):G285-92.

72 Senna-Fernandes V, França DL, de Souza D, Santos KC, Sousa RS, Manoel CV, et al. Acupuncture at "zusanli" (St.36) and "sanyinjiao" (SP.6) points on the gastrointestinal tract: a study of the bioavailability of $99 \mathrm{mTc}$-sodium pertechnetate in rats. Evid Based Complement Alternat Med. 2011;2011:823941.

73 de Jonge WJ, van den Wijngaard RM, The FO, ter Beek ML, Bennink RJ, Tytgat GN, et al. Postoperative ileus is maintained by intestinal immune infiltrates that activate inhibitory neural pathways in mice. Gastroenterology. 2003 Oct; 125(4):1137-47.

74 The FO, Boeckxstaens GE, Snoek SA, Cash JL, Bennink R, Larosa GJ, et al. Activation of the cholinergic anti-inflammatory pathway ameliorates postoperative ileus in mice. Gastroenterology. 2007 Oct;133(4):1219-28.

75 Munyaka P, Rabbi MF, Pavlov VA, Tracey KJ, Khafipour E, Ghia JE. Central muscarinic cholinergic activation alters interaction between splenic dendritic cell and CD4+CD25T cells in experimental colitis. PLoS One. 2014 Oct;9(10):e109272.

76 Stakenborg N, Labeeuw E, Gomez-Pinilla PJ, De Schepper S, Aerts R, Goverse G, et al. Preoperative administration of the 5-HT4 receptor agonist prucalopride reduces intestinal inflammation and shortens postoperative ileus via cholinergic enteric neurons. Gut. 2019 Aug;68(8):1406-16.

77 Stakenborg N, Wolthuis AM, Gomez-Pinilla PJ, Farro G, Di Giovangiulio M, Bosmans G, et al. Abdominal vagus nerve stimulation as a new therapeutic approach to prevent postoperative ileus. Neurogastroenterol Motil. 2017 Sep;29(9):e13075.

78 Hong GS, Pintea B, Lingohr P, Coch C, Randau T, Schaefer N, et al. Effect of transcutaneous vagus nerve stimulation on muscle activity in the gastrointestinal tract (transVa$\mathrm{Ga}$ ): a prospective clinical trial. Int J Colorectal Dis. 2019 Mar;34(3):417-22.

79 Zhang B, Xu F, Hu P, Zhang M, Tong K, Ma G, et al. Needleless Transcutaneous Electrical Acustimulation: A Pilot Study Evaluating Improvement in Post-Operative Recovery. Am J Gastroenterol. 2018 Jul;113(7):1026-35.

80 Ji H, Rabbi MF, Labis B, Pavlov VA, Tracey KJ, Ghia JE. Central cholinergic activation of a vagus nerve-to-spleen circuit alleviates experimental colitis. Mucosal Immunol. 2014 Mar;7(2):335-47.

81 Matteoli G, Gomez-Pinilla PJ, Nemethova A, Di Giovangiulio M, Cailotto C, van Bree SH, et al. A distinct vagal anti-inflammatory pathway modulates intestinal muscularis resident macrophages independent of the spleen. Gut. 2014 Jun;63(6):938-48.

82 Meroni E, Stakenborg N, Gomez-Pinilla PJ, De Hertogh G, Goverse G, Matteoli G, et al. Functional characterization of oxazolone-induced colitis and survival improvement by vagus nerve stimulation. PLoS One. 2018 May;13(5):e0197487.

83 Bonaz B, Sinniger V, Pellissier S. Vagus nerve stimulation: a new promising therapeutic tool in inflammatory bowel disease. J Intern Med. 2017 Jul;282(1):46-63.

84 Meregnani J, Clarençon D, Vivier M, Peinnequin A, Mouret C, Sinniger V, et al. Anti-inflammatory effect of vagus nerve stimulation in a rat model of inflammatory bowel disease. Auton Neurosci. 2011 Feb;160(1-2):82-9.

85 Sun P, Zhou K, Wang S, Li P, Chen S, Lin G, et al. Involvement of MAPK/NF- $\kappa B$ signaling in the activation of the cholinergic anti-inflammatory pathway in experimental colitis by chronic vagus nerve stimulation. PLoS One. 2013 Aug;8(8):e69424.

86 Bonaz B, Sinniger V, Hoffmann D, Clarençon D, Mathieu N, Dantzer C, et al. Chronic vagus nerve stimulation in Crohn's disease: a 6-month follow-up pilot study. Neurogastroenterol Motil. 2016 Jun;28(6):948-53.

87 Pott J, Hornef M. Innate immune signalling at the intestinal epithelium in homeostasis and disease. EMBO Rep. 2012 Aug; 13(8): 684-98.

88 Catalioto RM, Maggi CA, Giuliani S. Intestinal epithelial barrier dysfunction in disease and possible therapeutical interventions. Curr Med Chem. 2011;18(3):398-426.

89 Okumura R, Takeda K. Roles of intestinal epithelial cells in the maintenance of gut homeostasis. Exp Mol Med. 2017 May;49(5):e338. 
90 Van Landeghem L, Chevalier J, Mahé MM, Wedel T, Urvil P, Derkinderen P, et al. Enteric glia promote intestinal mucosal healing via activation of focal adhesion kinase and release of proEGF. Am J Physiol Gastrointest Liver Physiol. 2011 Jun;300(6):G97687.
91 Costantini TW, Krzyzaniak M, Cheadle GA, Putnam JG, Hageny AM, Lopez N, et al. Targeting $\alpha-7$ nicotinic acetylcholine receptor in the enteric nervous system: a cholinergic agonist prevents gut barrier failure after severe burn injury. Am J Pathol. 2012 Aug;181(2): $478-86$.
92 Hu S, Du MH, Luo HM, Wang H, Lv Y, Ma L, et al. Electroacupuncture at Zusanli (ST36) Prevents Intestinal Barrier and Remote Organ Dysfunction following Gut Ischemia through Activating the Cholinergic Anti-Inflammatory-Dependent Mechanism. Evid Based Complement Alternat Med. 2013;2013:592127. 